\title{
Néel's Magnetostatic Coupling in Sputtered Cu/Py/V/Py/MnIr/Cu Multilayers
}

\author{
M. UrbaniaK ${ }^{a, *}$, H. BrüCKL ${ }^{b}$, F. Stobiecki ${ }^{a}$, T. LUCiński ${ }^{a}$ \\ AND G. REISS ${ }^{b}$ \\ ${ }^{a}$ Institute of Molecular Physics, Polish Academy of Sciences \\ Smoluchowskiego 17, 60-179 Poznań, Poland \\ ${ }^{b}$ University of Bielefeld, Department of Physics \\ P.O. Box 100131, 33501 Bielefeld, Germany
}

(Received November 29, 2003)

\begin{abstract}
The correlation between magnetostatic or Néel's coupling and roughness of interfaces in sputtered $\mathrm{Cu} / \mathrm{Py} / \mathrm{V} / \mathrm{Py} / \mathrm{MnIr} / \mathrm{Cu}$ multilayers was investigated with the help of atomic force microscopy and magnetometry. It is shown that the coupling strongly depends on the roughness of seed $\mathrm{Cu}$ layer which in turn depends on sputtering power. Roughness levels off after deposition of consecutive layers to about $0.2 \mathrm{~nm} \mathrm{rms}$.
\end{abstract}

PACS numbers: 75.70.Cn, 68.65.Ac

\section{Introduction}

The coupling between ferromagnetic (F) layers in F/S/F multilayer structures ( $\mathrm{S}$ - non-magnetic spacer) influences very strongly magnetization reversal of the systems. In the so-called pinned spin valves ( $\mathrm{SVs}$ ), i.e. $\mathrm{AF} / \mathrm{F}_{1} / \mathrm{S} / \mathrm{F}_{2}$ structures, a direct contact between ferromagnetic layer and an antiferromagnet (AF) can lead to the appearance of a unidirectional anisotropy [1] with a shift field (exchange bias) $H_{\text {ex }}$ of up to dozens of $\mathrm{kA} / \mathrm{m}$. Indirect interaction, mediated by conduction electrons in spacer, can lead to an antiferromagnetic Ruderman-Kittel-KasuyaYoshida-like (RKKY-like) coupling between neighboring magnetic layers with saturation field of hundreds of $\mathrm{kA} / \mathrm{m}$. In some structures, especially in SVs in which

*corresponding author; e-mail: urbaniak@ifmpan.poznan.pl 
free, i.e. uncoupled to antiferromagnet, magnetic layer saturates in small magnetic fields another kind of coupling could be important. It is so-called magnetostatic (Néel's or orange peel) coupling which is caused by the interactions between discontinuities of magnetization ("magnetic charges") which form as a consequence of roughness of interacting $\mathbf{F}_{1}$ and $\mathbf{F}_{2}$ layers [2]. In many applications it is desirable to minimize this coupling to achieve the highest potential of the giant magnetoresistance and tunneling devices $[3-5]$. In this work we present the results of an investigation of such coupling in $\mathrm{Cu} / \mathrm{Py} / \mathrm{V} / \mathrm{Py} / \mathrm{MnIr}$ spin-valve-like system $\left(\mathrm{Py}=\mathrm{Ni}_{80} \mathrm{Fe}_{20}\right)$.

\section{Experimental}

The investigated multilayers of the $\mathrm{Si}(100) / 100 \mathrm{~nm}$ thermal Si-oxide/ $\mathrm{Cu}(\approx 20 \mathrm{~nm}) / \mathrm{Ni}_{80} \mathrm{Fe}_{20}\left(t_{\mathrm{Py} 1}\right) / \mathrm{V}\left(t_{\mathrm{V}}\right) / \mathrm{Ni}_{80} \mathrm{Fe}_{20}(4 \mathrm{~nm}) / \mathrm{Mn}_{83} \operatorname{Ir}_{17}(10 \mathrm{~nm}) / \mathrm{Cu}(3 \mathrm{~nm})$ structure, where $t_{\mathrm{Py} 1}=2-10 \mathrm{~nm}, t_{\mathrm{V}}=2.1-10.6 \mathrm{~nm}$, were sputtered with a base pressure of $8 \times 10^{-5} \mathrm{~Pa}$ and a process pressure $(\mathrm{Ar})$ of $0.2 \mathrm{~Pa}$. The roughness of the $\mathrm{Cu}$ seed layer was varied by changing a sputtering power $(P)$ from 93 to $250 \mathrm{~W}$, for other layers $93 \mathrm{~W}$ power was used. Exchange bias was induced in situ by a permanent magnet with a field of $9.5 \mathrm{kA} / \mathrm{m}$. Atomic force microscopy (AFM) was used to determine roughness of interfaces in the multilayer. Roughnesses were measured on the incomplete structures i.e. for example on top of $\mathrm{Si} / \mathrm{SiO} / \mathrm{Cu} / \mathrm{Py} / \mathrm{V}$ structure. In this work the roughness measured on a given incomplete structure is a measure of roughness of interface in a complete structure. Magnetic measurements were performed with vibrating sample magnetometer (VSM).

\section{Results and discussion}

A typical $M(H)$ dependence, like the one shown in Fig. 1, allowed us to determine shift fields $H_{S}$ of the studied samples. As can be seen, the "soft" loop of the free Py1 layer (see the inset of Fig. 1) is shifted in the negative field direction (i.e. the same as the loop of the top, pinned Py2 layer, see Fig. 2) which indicates the ferromagnetic coupling between Py layers. Within the resolution of our measurements the observed coupling was always ferromagnetic although in many cases, for higher $t_{\mathrm{V}}$ values, barely detectable. The maximum $H_{\mathrm{S}}$ values recorded did not exceed $0.9 \mathrm{kA} / \mathrm{m}$. We cannot unambiguously separate different contributions to the overall coupling [6] but since the fall off of $H_{\mathrm{S}}$ with increasing spacer thickness $t_{\mathrm{S}}$ is fairly well described by a $\exp \left(-t_{\mathrm{S}}\right)$ dependence (see Eq. (1)) we believe that a possible bridging of the Py layers through vanadium layer plays only minor role, if any, in our samples.

AFM measurements show that the Cu seed layer roughness, $r$, depends markedly on sputtering power used (Fig. 3). In general rms roughnesses determined from AFM scans are low and never exceed $0.5 \mathrm{~nm}$. The roughnesses on top 


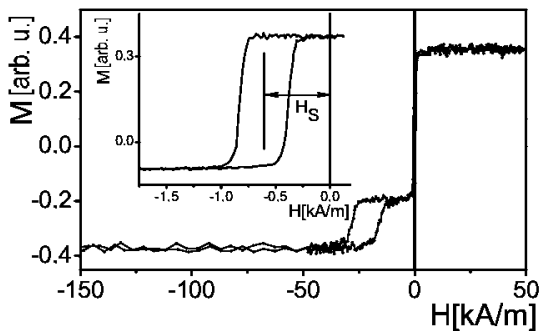

Fig. 1. Exemplary $M(H)$ dependence for the $\operatorname{Si}(100) / 100 \mathrm{~nm}$ thermal Si-oxide $/ \mathrm{Cu}(\approx 20 \mathrm{~nm}) / \mathrm{Ni}_{80} \mathrm{Fe}_{20}(10 \mathrm{~nm}) / \mathrm{V}(2.1 \mathrm{~nm}) / \mathrm{Ni}_{80} \mathrm{Fe}_{20}(4 \mathrm{~nm}) / \mathrm{Mn}_{83} \mathrm{Ir}_{17}(10 \mathrm{~nm}) / \mathrm{Cu}(3 \mathrm{~nm})$ structure. Inset shows the minor hysteresis loop with an appropriate $H_{\mathrm{S}}$ value.

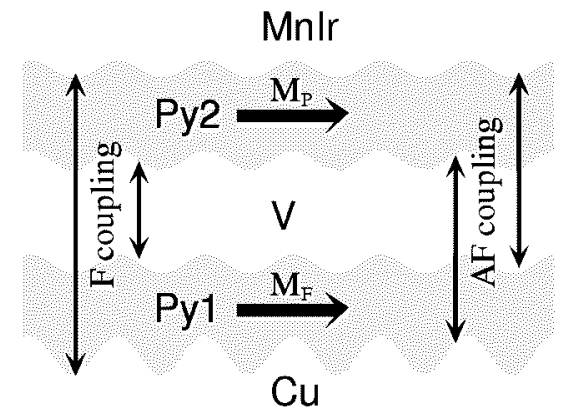

Fig. 2. A schematic view of the magnetic part of investigated multilayers. Ferromagnetic and antiferromagnetic magnetostatic interactions between different pairs of interfaces are also shown.

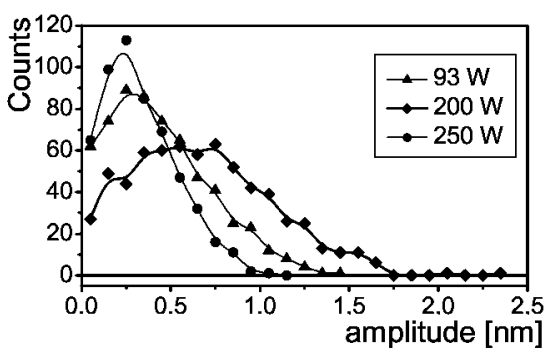

Fig. 3. Sputtering power dependence of histograms showing the distribution of roughness amplitudes (see the text) on top of the Cu layers in the $\mathrm{Si}(100) / 100 \mathrm{~nm}$ thermal Si-oxide/Cu $(\approx 20 \mathrm{~nm})$ structure. To extract the heights the consecutive line scans of AFM image were digitally smoothed with low pass filter and the heights of all local maxima and minima were recorded [7].

of $\mathrm{Cu}$ and $\mathrm{V}$ are isotropic (directions 45,90 , and $135^{\circ}$ relative to the scan direction were checked). For $200 \mathrm{~W}$ sputtering power roughness of $\mathrm{Cu}$ seed layer attains a local maximum (Fig. 4) and then decreases to value lower than in the samples de- 


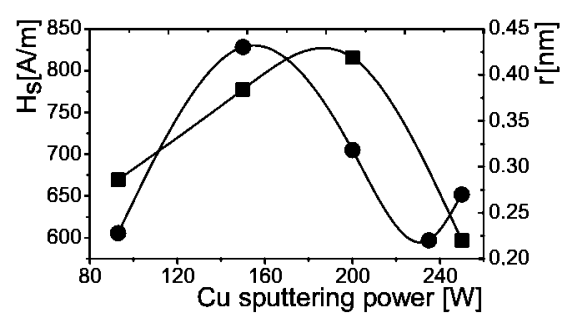

Fig. 4. Sputtering power dependence of shift field $H_{\mathrm{S}}$ for $\operatorname{Si}(100) / 100 \mathrm{~nm}$ thermal Si-oxide $/ \mathrm{Cu}(\approx 20 \mathrm{~nm}) / \mathrm{Ni}_{80} \mathrm{Fe}_{20}(6 \mathrm{~nm}) / \mathrm{V}(2.1 \mathrm{~nm}) / \mathrm{Ni}_{80} \mathrm{Fe}_{20}(4 \mathrm{~nm}) / \mathrm{Mn}_{83} \operatorname{Ir}_{17}(10 \mathrm{~nm}) /$ $\mathrm{Cu}(3 \mathrm{~nm})$ samples $(0)$ and AFM measured rms roughness $r$ for $\mathrm{Si}(100) / 100 \mathrm{~nm}$ thermal Si-oxide $/ \mathrm{Cu}(\approx 20 \mathrm{~nm})$ samples $(\square)$.

posited at $P=93 \mathrm{~W}$. Figure 3 shows the appropriate histograms [7] each of which was obtained from two sets of five neighboring $1 \mu \mathrm{m}$ AFM scans. Although there is a considerable spread of roughness amplitudes one sees that surfaces of films deposited with 93 and $250 \mathrm{~W}$ have similar height distribution. This similarity is reflected in $H_{\mathrm{S}}$ dependence on the sputtering power (Fig. 4) where one sees that shift fields have similar values for both samples, too.

The maxima of the $r(P)$ and $H_{\mathrm{S}}(P)$ dependences are mutually shifted, though. Nevertheless, there seems to be a direct correlation between the $\mathrm{Cu}$ underlayer roughness as obtained from AFM measurements and the shift field $H_{\mathrm{S}}$. We believe that it corroborates our assumption of magnetostatic origin of shift of the hysteresis loop of the free Py layer since in theoretical expressions for $H_{\mathrm{S}}$ value $[2,8]$ shift field is a linear function of roughness of every individual layer.

As shown, the AFM measurements demonstrate that the roughness of $\mathrm{Cu}$ seed layer clearly depends on $P$. However, the roughnesses of consecutive interfaces seem to be almost independent of the roughness of $\mathrm{Cu} / \mathrm{Py} 1$ interface. After a deposition of $2 \mathrm{~nm}$ of Py on top of Cu layer, rms roughness diminishes from 0.3 to $0.16 \mathrm{~nm}$ and changes only a little after the deposition of a consecutive $8 \mathrm{~nm}$ of Py. The vanadium layer essentially does not influence the roughness, too. We conclude that in our specific structure only roughness of $\mathrm{Cu}$ seed layer is seriously influenced by the initial sputtering conditions and that the consecutive layers smoothen it to about $0.2 \mathrm{~nm}$. This behavior is not obligatory, since for layers deposited with $\mathrm{Cu}$ sputtering power of $150 \mathrm{~W}$ roughness slightly increases after deposition of $6 \mathrm{~nm}$ of Py in comparison to $2 \mathrm{~nm}$ Py overlayer.

As seen in Fig. 5, roughness wavelength, $\lambda$, seems to be very similar on $\mathrm{Cu}$ and $\mathrm{V}$ upper interfaces giving justification to the assumption of a conformal growth of our multilayers [8].

To estimate the shift field $H_{\mathrm{S}}$ values we used the known Néel formula for magnetostatic coupling $[2,9]$ :

$$
H_{\mathrm{S}}=\frac{\pi^{2}}{\sqrt{2}}\left(\frac{h^{2}}{\lambda t_{\mathrm{f}}}\right) M_{\mathrm{p}} \mathrm{e}^{-2 \pi \sqrt{2} t_{\mathrm{S}} / \lambda}
$$




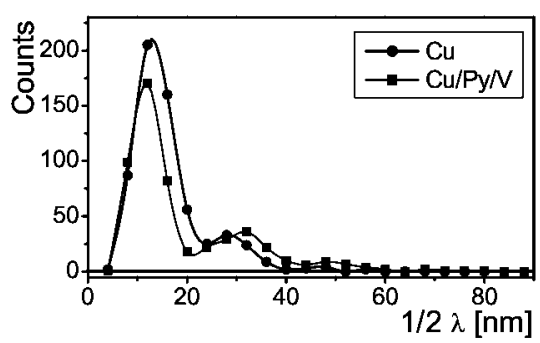

Fig. 5. Histograms indicating the distribution of wavelengths of surface profiles of $\mathrm{Cu}(0)$ and $\mathrm{Cu} / \mathrm{Py}(10 \mathrm{~nm}) / \mathrm{V}(2 \mathrm{~nm})(\square)$ samples obtained with $93 \mathrm{~W}$ sputtering power. To extract wavelengths the consecutive line scans of AFM image were smoothed with low pass filter and the positions of all local maxima and minima were recorded [7].

where $h$ denotes roughness amplitude, $t_{\mathrm{f}}$ - thickness of the free layer, $t_{\mathrm{S}}$ non-magnetic spacer thickness and $M_{\mathrm{p}}$ - the magnetization of the pinned layer. Originally Néel derived his formula for two interacting semi-infinite magnetic layers and included thus in analysis only two rough surfaces [2]. It was shown later [8] that the above description can be refined to successfully describe interactions of two thin ferromagnetic films. In such case one has to include four interactions between four pairs of interfaces (see Fig. 2) i.e. in our case ferromagnetic couplings between the top interface of Py1 layer and the bottom of Py2 and the bottom interface of Py1 layer and the top interface of Py2 layer and antiferromagnetic couplings between respectively top and bottom interfaces of both layers. To estimate the coupling field $H_{\mathrm{S}}$ we used roughness values obtained from AFM scans with the assumption that roughness on both interfaces of Py2 layer is the same. We believe that the relative independence of roughnesses of the upper interfaces on Cu seed layer roughness justifies this assumption i.e. taking measured roughness of $\mathrm{V}$ layer as the estimate of the Py2 layer upper roughness. We assumed also that interfaces are correlated [8]. It should be noted that as rms of sinusoid is $\sqrt{2}$ times smaller than its amplitude we multiplied rms roughness obtained from AFM by that factor prior to calculating $H_{\mathrm{S}}$ with Eq. (1). Our calculated values of coupling field are the algebraic sums of the four previously mentioned interactions [2, 8]. For the ferromagnetic interaction between the bottom surface of Py1 layer (roughness $h_{1}$ ) and the top surface of the Py2 layer (roughness $h_{4}$ ) (see Fig. 2) the expression (1) takes the form

$$
H_{\mathrm{S}}=\frac{\pi^{2}}{\sqrt{2}}\left(\frac{h_{1} h_{4}}{\lambda t_{\mathrm{Py} 1}}\right) M_{\mathrm{p}} \mathrm{e}^{-2 \pi \sqrt{2}\left(t_{\mathrm{Py} 1}+t_{\mathrm{V}}+t_{\mathrm{Py} z}\right) / \lambda} .
$$

The model values of $H_{\mathrm{S}}$ are shown in Fig. 6 together with respective experimental dependence.

The correspondence between the model values and the experiment is satisfactory keeping in mind simplifying assumptions made. It should be noted in view 


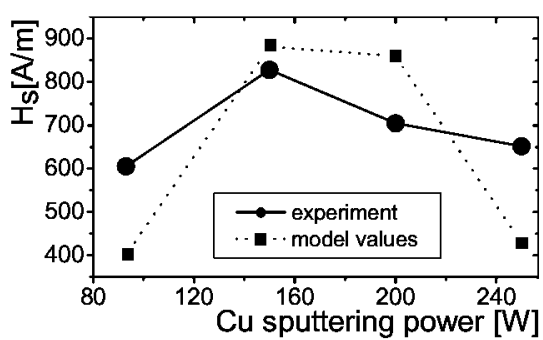

Fig. 6. Sputtering power dependence of shift field $H_{\mathrm{S}}$ for $\operatorname{Si}(100) / 100 \mathrm{~nm}$ thermal Si-oxide/Cu( $\approx 20 \mathrm{~nm}) / \mathrm{Ni}_{80} \mathrm{Fe}_{20}(6 \mathrm{~nm}) / \mathrm{V}(2.1 \mathrm{~nm}) / \mathrm{Ni}_{80} \mathrm{Fe}_{20}(4 \mathrm{~nm}) / \mathrm{Mn}_{83} \operatorname{Ir}_{17}(10 \mathrm{~nm}) /$ $\mathrm{Cu}(3 \mathrm{~nm})$ samples $(0)$ and model values obtained with Néel's expression ( $\square$ ) (compare Fig. 4).

of dependence shown in Fig. 4 that according to the model the $\mathrm{Cu}$ seed layer roughness can both increase and decrease $H_{S}$ depending on the ratio of roughnesses on both sides of the upper Py layer. Although the Néel model is rather simple it was already shown that it can reasonably well describe experimental results $[8,10,11]$.

We believe that the main source of error in our estimate is the determination of roughness modulation wavelength. We assumed, based on the dependence of Fig. 5, that $\lambda$ is about $25 \mathrm{~nm}$. One can see in Fig. 7 that $H_{\mathrm{S}}$ depends very much on $\lambda$ value and any error in its determination causes serious changes in obtained coupling value. The assumption of the conformal growth may be a source of error, too. If roughnesses of interacting interfaces are out of phase the coupling field value could even change sign [5]. It should be expected that a better estimation of effective roughness wavelength of morphologically complicated interfaces is needed to improve correspondence with experiment.

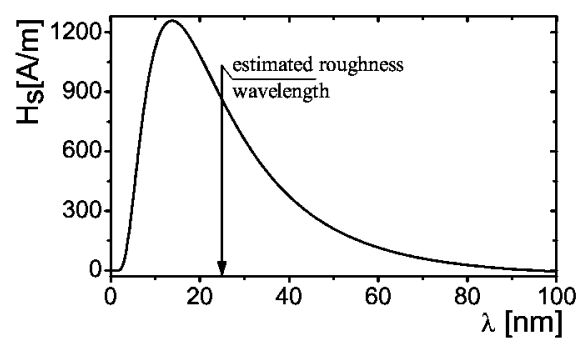

Fig. 7. Model $\lambda$ dependence of the shift field $H_{S}$ for $\operatorname{Si}(100) / 100 \mathrm{~nm}$ thermal Si-oxide/Cu( $\approx 20 \mathrm{~nm}) / \mathrm{Ni}_{80} \mathrm{Fe}_{20}(6 \mathrm{~nm}) / \mathrm{V}(2.1 \mathrm{~nm}) / \mathrm{Ni}_{80} \mathrm{Fe}_{20}(4 \mathrm{~nm}) / \mathrm{Mn}_{83} \operatorname{Ir}_{17}(10 \mathrm{~nm}) /$ $\mathrm{Cu}(3 \mathrm{~nm})$ multilayer obtained with $200 \mathrm{~W} \mathrm{Cu}$ sputtering power. Lambda value estimated from dependence of Fig. 5 is also shown. 


\section{Conclusions}

We have investigated magnetostatic (Néel's) coupling in the $\mathrm{Si}(100) / 100 \mathrm{~nm}$ thermal Si-oxide $/ \mathrm{Cu}(\approx 20 \mathrm{~nm}) / \mathrm{Ni}_{80} \mathrm{Fe}_{20}\left(t_{\mathrm{Py} 1}\right) / \mathrm{V}\left(t_{\mathrm{V}}\right) / \mathrm{Ni}_{80} \mathrm{Fe}_{20}(4 \mathrm{~nm}) / \mathrm{Mn}_{83} \mathrm{Ir}_{17}$ $(10 \mathrm{~nm}) / \mathrm{Cu}(3 \mathrm{~nm})$ structure. It was shown that the $\mathrm{Cu}$ seed layer roughness decisively influences the coupling field in the multilayer. It was also shown that Néel's formula allowed us to approximate measured values of $H_{\mathrm{S}}$ but there remains a considerable discrepancy with the experimental results probably due to our inability to properly characterize the interface morphology. From the application point of view the strong dependence of coupling field value on $\mathrm{Cu}$ sputtering power should be kept in mind when aiming at low values of $H_{\mathrm{S}}$.

\section{Acknowledgments}

This work was supported by a Polish-German Scientific and Technological Cooperation Joint Project POL 99/023.

\section{References}

[1] J. Nogues, I.K. Schuller, J. Magn. Magn. Mater. 192, 203 (1999).

[2] T.C. Schulthess, W.H. Butler, J. Appl. Phys. 87, 5759 (2000).

[3] D. Wang, J.M. Daughton, D. Reed, W.D. Wang, J.-Q. Wang, IEEE Trans. Magn. 36, $2802(2000)$

[4] M. Tondra, J.M. Daughton, D. Wang, R. Beech, A. Fink, J.A. Taylor, J. Appl. Phys. 83, 6688 (1998).

[5] J. Zhang, R.M. White, IEEE Trans. Magn. 32, 4630 (1996).

[6] H.D. Chopra, B.J. Hockey, P.J. Chen, W.F. Egelhoff, Jr., M. Wuttig, S.Z. Hua, Phys. Rev. B 55, 8390 (1997).

[7] B.D. Schrag, A. Anguelouch, S. Ingvarsson, Gang Xiao, Yu Lu, P.L. Trouilloud, A. Gupta, R.A. Wanner, W.J. Gallagher, P.M. Rice, S.S.P. Parkin, Appl. Phys. Lett. 77, $2373(2000)$.

[8] J.C.S. Kools, W. Kula, D. Mauri, Tsann Lin, J. Appl. Phys. 85, 4466 (1999).

[9] J.C.S. Kools, J. Appl. Phys. 77, 2993 (1995).

[10] J.C.S. Kools, A.J. Devasahayam, K. Rook, Chih-Ling Lee, M. Mao, J. Appl. Phys. 93, 7921 (2003).

[11] T. Luciński, A. Hütten, H. Brückl, T. Hempel, S. Heitmann, G. Reiss, Phys. Status Solidi A 196, 97 (2003). 